\title{
THE STABILITY OF THE SINE EQUATION
}

\author{
PIOTR W. CHOLEWA
}

\begin{abstract}
Let $\delta$ be a positive real constant and let $G$ be an abelian group (written additively) in which division by 2 is uniquely performable. Every unbounded complex-valued function $f$ on $G$ satisfying the inequality
\end{abstract}

$$
\left|f(x+y) f(x-y)-f(x)^{2}+f(y)^{2}\right| \leqslant \delta \quad \text { for all } x, y \in G
$$

has to be a solution of the sine functional equation

$$
f(x+y) f(x-y)=f(x)^{2}-f(y)^{2} \text { for all } x, y \in G .
$$

A stability problem in functional equation theory seems to have been first raised by S. M. Ulam in [4] and D. H. Hyers who proved the stability of a linear functional equation (see [3]). Generalizations of this result appeared then in many papers. In 1979 J. A. Baker, J. Lawrence and F. Zorzitto showed in [2] that the equation $f(x+y)=f(x) f(y)$ has a different kind of stability. Exactly, they proved that if $f$ is a real-valued function defined on a rational vector space $V$ and satisfies the inequality $|f(x+y)-f(x) f(y)| \leqslant \delta$ for all $x, y \in V$ and some real $\delta>0$, then either $f$ is bounded or $f(x+y)=f(x) f(y)$ for all $x, y \in V$. This result was then generalized by J. A. Baker (see [1]) who also had obtained a similar result for the cosine equation $f(x+y)+f(x-y)=2 f(x) f(y)$. The main purpose of this paper is to prove an analogous result for the sine functional equation

$$
f(x+y) f(x-y)=f(x)^{2}-f(y)^{2} .
$$

We shall start from some general assumptions. Let $G$ be an abelian group (written additively) in which division by 2 is uniquely performable (i.e. for each $x \in G$ there exists a unique $y \in G$ such that $y+y=x$; such a $y$ will be denoted by $x / 2$ ). Let $f$ be a complex-valued function defined on $G$ and such that the inequality

$$
\left|f(x+y) f(x-y)-f(x)^{2}+f(y)^{2}\right| \leqslant \delta
$$

holds for all $x, y \in G$ and some real $\delta>0$. Moreover, assume that $f$ is an unbounded function.

For the proof we need the following three lemmas.

LEMMA 1.

$$
f(0)=0 .
$$

Received by the editors August 9, 1982.

1980 Mathematics Subject Classification. Primary 39B40; Secondary 39B70, 39C05.

Key words and phrases. Functional equation, sine equation, stability.

(C)1983 American Mathematical Society $0002-9939 / 82 / 0000-1314 / \$ 01.75$ 
Proof. Put $x=y$ and $u=2 x$ in inequality (2). Then

$$
\delta \geqslant\left|f(u) f(0)-f(u / 2)^{2}+f(u / 2)^{2}=\right| f(u)|| f(0) \mid
$$

and since $|f(u)|$ can be as large as desired, we must have

$$
f(0)=0 . \quad \text { Q.E.D. }
$$

Now put $x=(u+v) / 2, y=(u-v) / 2$ in (2). Then $x+y=u, x-y=v$ and inequality (2) assumes the form

$$
\left|f(u) f(v)-f\left(\frac{u+v}{2}\right)^{2}+f\left(\frac{u-v}{2}\right)^{2}\right| \leqslant \delta \quad \text { for all } u, v \in G .
$$

Since $f$ is an unbounded function, there exists an $a \in G$ such that $|f(a)| \geqslant 4$. Let $g: G \rightarrow \mathbf{C}$ (C denotes here the field of all complex numbers) be the function defined by

$$
g(x)=\frac{f(x+a)-f(x-a)}{2 f(a)} \quad \text { for all } x \in G .
$$

LEMMA 2. For all $x, y \in G$ the following inequality is satisfied:

$$
|f(x+y)+f(x-y)-2 f(x) g(y)| \leqslant \delta .
$$

Proof. Let $x, y \in G$. Using (4) and (5) we get

$$
\begin{aligned}
\mid f(x+y)+ & f(x-y)-2 f(x) g(y) \mid \\
= & \frac{1}{|f(a)|}|f(x+y) f(a)+f(x-y) f(a)-2 f(a) f(x) g(y)| \\
\leqslant & \frac{1}{|f(a)|}\left|f(x+y) f(a)-f\left(\frac{x+y+a}{2}\right)^{2}+f\left(\frac{x+y-a}{2}\right)^{2}\right| \\
& +\frac{1}{|f(a)|}\left|f(x-y) f(a)-f\left(\frac{x-y+a}{2}\right)^{2}+f\left(\frac{x-y-a}{2}\right)^{2}\right| \\
& +\frac{1}{|f(a)|}\left|f\left(\frac{x+y+a}{2}\right)^{2}-f\left(\frac{x-y-a}{2}\right)^{2}-f(x) f(y+a)\right| \\
& +\frac{1}{|f(a)|}\left|f(x) f(y-a)-f\left(\frac{x+y-a}{2}\right)^{2}+f\left(\frac{x-y+a}{2}\right)^{2}\right| \\
& +\left|2 f(x) \frac{f(y+a)-f(y-a)}{2 f(a)}-2 f(x) g(y)\right| \\
\leqslant & \frac{4 \delta}{|f(a)|} \leqslant \delta . \quad \text { Q.E.D. }
\end{aligned}
$$

LEMMA 3. The following equation is satisfied for all $x, y \in G$ :

$$
f(x+y)+f(x-y)=2 f(x) g(y) .
$$


Proof. Let $x, y$ be two arbitrarily fixed points of $G$. Then, using (4) and (6), for all $z \in G$ we have

$$
\begin{aligned}
&|f(z)||f(x+y)+f(x-y)-2 f(x) g(y)| \\
&=|f(z) f(x+y)+f(z) f(x-y)-2 f(x) f(z) g(y)| \\
& \leqslant\left|f(z) f(x+y)-f\left(\frac{z+x+y}{2}\right)^{2}+f\left(\frac{z-x-y}{2}\right)^{2}\right| \\
&+\left|f(z) f(x-y)-f\left(\frac{z+x-y}{2}\right)^{2}+f\left(\frac{z-x+y}{2}\right)^{2}\right| \\
&+\left|f\left(\frac{z+x+y}{2}\right)^{2}-f\left(\frac{z-x+y}{2}\right)^{2}-f(z+y) f(x)\right| \\
&+\left|f\left(\frac{z+x-y}{2}\right)^{2}-f\left(\frac{z-x-y}{2}\right)^{2}-f(z-y) f(x)\right| \\
&+|[f(z+y)+f(z-y)] f(x)-2 f(z) g(y) f(x)| \\
& \leqslant 4 \delta+\delta|f(x)|=\delta(4+|f(x)|) .
\end{aligned}
$$

Therefore

$$
|f(x+y)+f(x-y)-2 f(x) g(y)| \leqslant(4+|f(x)|) \delta /|f(z)|,
$$

and since $f$ is assumed to be an unbounded function and $x$ is a fixed element, $(4+|f(x)|) \delta /|f(z)|$ is as small as we wish. Hence we must have

$$
|f(x+y)+f(x-y)-2 f(x) g(y)|=0,
$$

whence

$$
f(x+y)+f(x-y)=2 f(x) g(y) .
$$

Since $x$ and $y$ were arbitrarily fixed elements of $G$, equation (7) holds for all $x, y \in G$. Q.E.D.

Now we are able to prove the main result of our paper.

THEOREM. Every unbounded function $f: G \rightarrow \mathbf{C}$ satisfying inequality

$$
\left|f(x+y) f(x-y)-f(x)^{2}+f(y)^{2}\right| \leqslant \delta \quad \text { for all } x, y \in G
$$

has to be a solution of the equation

$$
f(x+y) f(x-y)=f(x)^{2}-f(y)^{2} .
$$

Proof. Let $f$ be an unbounded solution of inequality (2). Put $x=0$ in (7). Then, using (3), we obtain

$$
f(y)+f(-y)=0 \text { for all } y \in G,
$$

i.e.

$$
f(y)=-f(-y) \text { for all } y \in G .
$$

Now, put $x=(u+v) / 2, y=(u-v) / 2$ in (7). Hence

$$
f(u)+f(v)=2 f\left(\frac{u+v}{2}\right) g\left(\frac{u-v}{2}\right) \text { for all } u, v \in G .
$$


Then, from (3) and (9), we infer that

$$
f(x+y)=f(x+y)+f(0)=2 f\left(\frac{x+y}{2}\right) g\left(\frac{x+y}{2}\right) \text { for all } x, y \in G,
$$

and

$$
f(x-y)=f(x-y)+f(0)=2 f\left(\frac{x-y}{2}\right) g\left(\frac{x-y}{2}\right) \text { for all } x, y \in G .
$$

Using (8) and (9) we also have

$$
f(x)-f(y)=f(x)+f(-y)=2 f\left(\frac{x-y}{2}\right) g\left(\frac{x+y}{2}\right) \text { for all } x, y \in G .
$$

Now, using (9)-(12) we obtain

$$
\begin{aligned}
f(x+y) f(x-y) & =\left[2 f\left(\frac{x+y}{2}\right) g\left(\frac{x+y}{2}\right)\right]\left[2 f\left(\frac{x-y}{2}\right) g\left(\frac{x-y}{2}\right)\right] \\
& =\left[2 f\left(\frac{x+y}{2}\right) g\left(\frac{x-y}{2}\right)\right]\left[2 f\left(\frac{x-y}{2}\right) g\left(\frac{x+y}{2}\right)\right] \\
& =[f(x)+f(y)][f(x)-f(y)]=f(x)^{2}-f(y)^{2}
\end{aligned}
$$

for all $x, y \in G$. Q.E.D.

REMARK 1. J. A. Baker proved in [1] that, concerning the stability of the cosine equation, a function $g$ satisfying inequality

$$
|g(x+y)+g(x-y)-2 g(x) g(y)| \leqslant \delta
$$

is either a solution of the cosine equation or it is bounded by a constant depending on $\delta$ only. The crucial step of the proof was that the function satisfying the above inequality is either bounded by such a constant or unbounded. It is not the case for the sine equation; indeed, the (bounded) functions

$$
f_{N}(x)=N \sin x+1 / N
$$

satisfy inequality (2) with $\delta=3$,

$$
\left|f_{N}(x+y) f_{N}(x-y)-f_{N}(x)^{2}+f_{N}(y)^{2}\right| \leqslant 3
$$

for all real numbers $x, y$ and all positive integers $N$. Nevertheless, for each positive real number $M$, the inequality $\left|f_{N}(x)\right| \leqslant M$ fails to hold for certain $x$ and $N$.

REMARK 2. We must emphasize that our main result is proved for complex-valued functions only. Essential difficulties appear while considering functions with values in the algebra of quaternions or Cayley numbers, despite the multiplicativity of the norm; associativity, as well as commutativity, was necessary for us to prove our theorem.

\section{REFERENCES}

1. J. A. Baker, The stability of the sine equation, Proc. Amer. Math. Soc. 80 (1980), 411-416.

2. J. A. Baker, J. Lawrence and F. Zorzitto, The stability of the equation $f(x+y)=f(x) f(y)$, Proc. Amer. Math. Soc. 74 (1979), 242-246.

3. D. H. Hyers, On the stability of the linear functional equation, Proc. Nat. Acad. Sci. U.S.A. 27 (1941), 222-224.

4. S. M. Ulam, A collection of mathematical problems, Interscience, New York, 1960.

Institute of Mathematics, Silesian University, Bankowa 14, 40-007 Katowice, Poland 